\title{
Strut-and-tie model for the support of steel beams crossing concrete beams
}

\author{
F. Marie ${ }^{a *}$ and H. Somja ${ }^{a}$ \\ ${ }^{a}$ National institute of applied sciences, Department of Civil Engineering, Rennes, France \\ *corresponding author, e-mail address: frederic.marie@insa-rennes.fr
}

\begin{abstract}
Steel and concrete construction can still be regarded as two distinct industrial sectors leading to separated design procedures. Even steel-concrete composite buildings remain designed as steel structures, with a limited benefit of the presence of concrete slabs.

For some years however, a more integrated design between both materials is investigated. It tries to combine them in order to take advantage from their respective qualities : the high resistance of the steel on one hand and the low cost and good fire resistance of the concrete on the other hand, for example. One of the advantages of the concrete is also the easiness in the fabrication of joints, thanks to the monolithic nature of the concrete cast in place, whereas the metallic joints by bolting or welding ask for more technical work, and represent a non-negligible part of the cost of a structure. It is therefore rather natural, in a hybrid concrete-steel conception, to try to use this advantage of the concrete.

In this context, this article presents a work that was made in the RFCS SMARTCOCO project. It focuses on the design of the support of a steel secondary beam crossing a primary beam in concrete, by simple direct contact. On the basis of an experimental campaign comprising five full-scale tests, the angle of diffusion of the forces and the distribution of the stresses in the stirrups are studied and a specific strut-and-tie model is developed. Specimens of this campaign consist of a simply supported concrete beam crossed in its middle by a steel profile, with or without stiffeners, loaded by two jacks, one at each end of the steel profile.

First the experimental campaign is described. Then, internal stresses are compared with the predictions of a strut and tie model deduced from elastic stress trajectories. Finally, simplified design guidance is deduced.
\end{abstract}

Keywords: Hybrid concrete-steel structures; indirect support; strut-and-tie model.

\section{Introduction}

In usual concrete construction, large spans for beams sustaining slabs can hardly be attained without having recourse to prestressing or poststressing techniques if beam heights are limited. Prestressing implies on one hand the prefabrication of the beams, that are heavy and therefore difficult to set up, and on the other hand complications in the connections, when reinforcing bars sticking out the beam cross the rebars of the parts cast in place. Post stressing leads to complications on site, as specific equipment has to be used for stressing operations.
In such a context, steel members can be an alternative, as they are quite lightweight and reach the same mechanical performances than stressed concrete members. However their connection to the rest of the structure is not handled in present codes whereas it rises specific problems.

In this paper, a work that handled the particular point of secondary steel beams crossing primary concrete beams is presented, see Fig. 1. This case is handled in Eurocode 2 under the name "indirect support" when the two series of beams are made of concrete. This work is a part of the RFCS SMARTCOCO project that was intended to fill gaps in knowledge and 
provide design guidance for steel profiles used within reinforced concrete buildings.

First the experimental campaign is described. Then, internal stresses are compared with the predictions of a strut and tie model deduced from elastic stress trajectories. Finally, simplified design guidance is deduced.

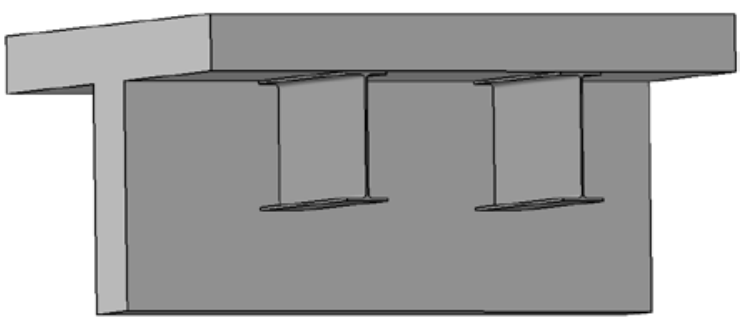

Fig. 1. Indirect support of steel beams supporting a slab.

\section{Experimental tests}

\subsection{Test specimens and test setup}

The specimens consist of a concrete beam crossed in its middle by a steel profile, see Fig. 2. The load is applied to the steel profile by 2 jacks, one at each end of the steel profile. Moreover a horizontal reaction is applied at each end of the specimen in order to increase the capacity of the concrete beam.

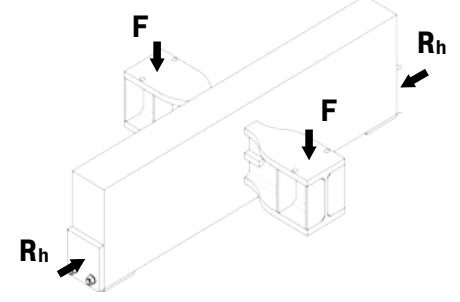

Fig. 2. Specimen and loading.

5 tests are carried out, with two different configurations :

- 2 specimens with a HE340M profile, named CS-H;

- 3 specimens with a HE340M profile with median horizontal stiffeners, named CS$\mathrm{H}+\mathrm{R}$.

The width of the steel flanges has been reduced in the embedded part of the steel beam, in order to limit the resistance in the investigated zone. Main dimensions are given Fig. 3. The dimensions of the steel section are given Fig. 4, and the rebars arrangement Fig. 5.

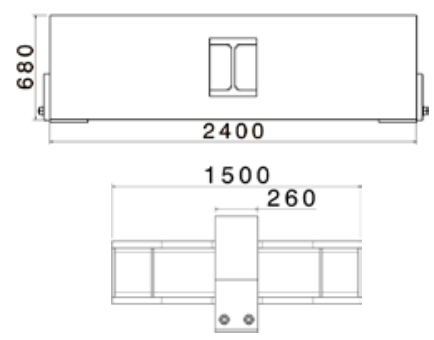

Fig. 3. Main dimensions of the specimen.

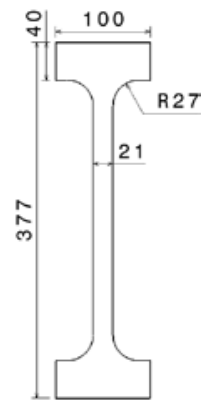

CS-H

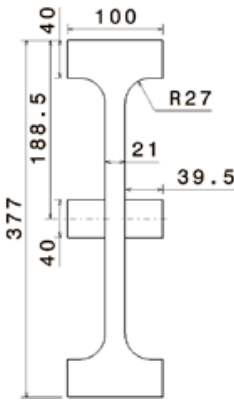

$\mathrm{CS}-\mathrm{H}+\mathrm{R}$

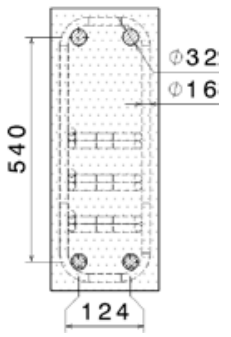

concrete
Fig. 4. Dimensions of the steel and concrete sections.

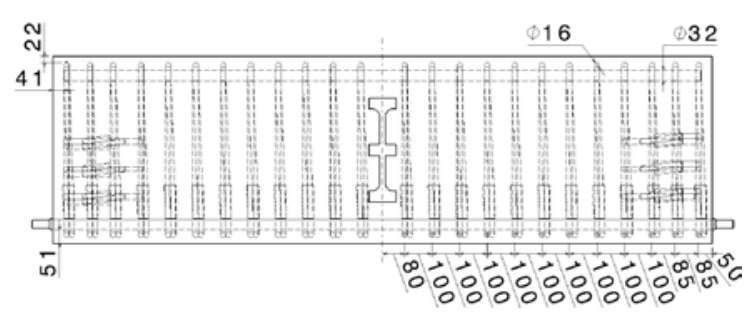

Fig. 5. Rebar arrangement.

\subsection{Experimental results}

The load-displacement curves are presented on Fig. 6. As can be seen, specimens with or without transverse stiffeners behave the same way. The differences observed for CS-H-1 and CS-H+R-3 are merely due to variations in the horizontal reaction effectively applied on the specimen, see Fig.7.

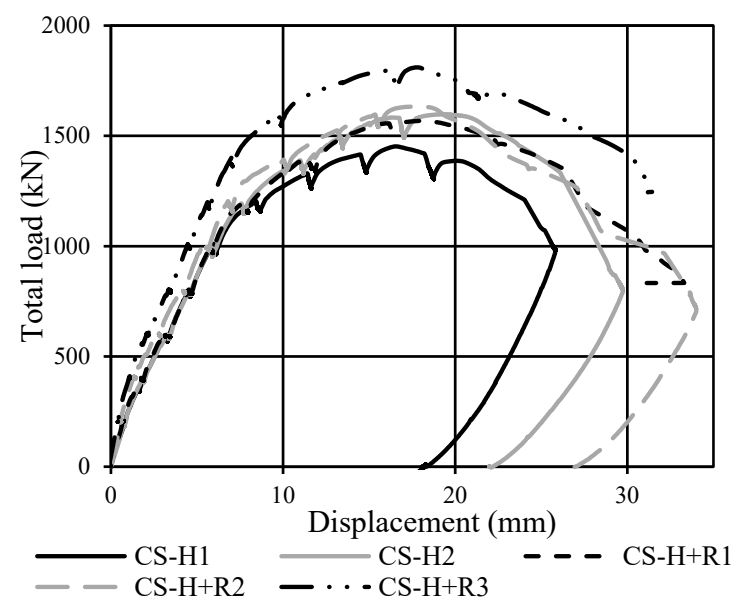

Fig. 6. Vertical force-displacement curves. 


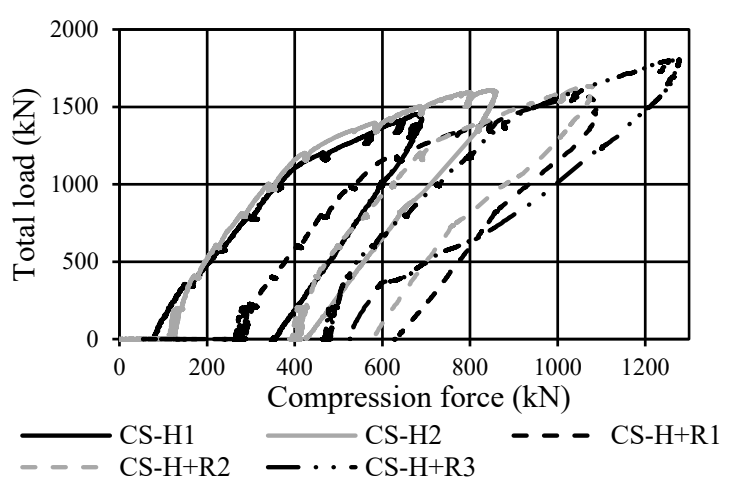

Fig. 7. Horizontal force-vertical force curves.

The same behavior has been observed for all the specimens. The first kind of cracking to develop is a bending one : vertical cracks appear beneath the steel profile. Next, shear cracks with an orientation of about $45^{\circ}$, starting from the steel profile and going to the bottom side of the specimen, develop. For a load of about $1200 \mathrm{kN}$, vertical cracks beneath the steel profile open largely, showing that the longitudinal bars yield. This is confirmed by the evolution of the strains measured by strain gauges. Anyway this first yielding does not lead to the full collapse of the specimen, as the horizontal support allows the development of an arch effect in the specimen. A maximum load ranging from 1400 to $1800 \mathrm{kN}$ is attained in the different specimens.

After reaching the maximum, a punching cone appears and the test is stopped when the first stirrup breaks. Fig. 8 shows the specimen CS$\mathrm{H}+\mathrm{R} 3$ after collapse.

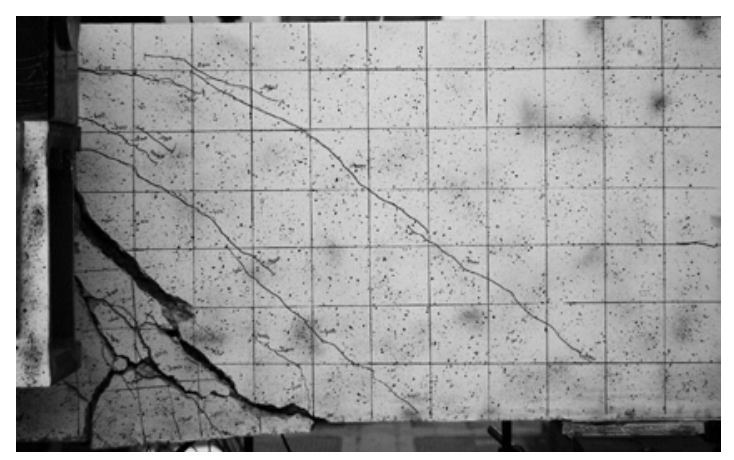

Fig. 8. Specimen CSH-R+3 after collapse.

The evolution of the stresses in the stirrups of the specimen CHS-R+3 is shown in Fig. 9. As in other specimens, it has been observed that only the first stirrups near the steel profile are yielded. This invalidates the model that was considered in the preliminary design, with parallel struts starting from the steel profile with an angle of $45^{\circ}$, and mobilizing the yielding of 5 stirrups, see Fig. 10. The analysis of the forces even shows that the steel profile is suspended only by 4 stirrups on each side.
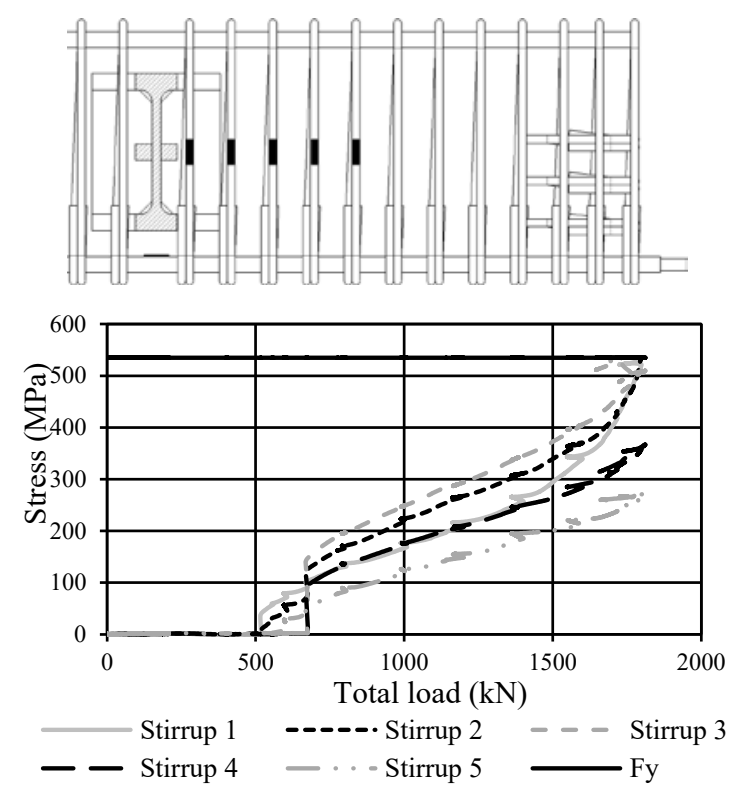

Fig. 9. Stresses in the stirrups : specimen $\mathrm{CSH}$ $\mathrm{R}+3$.

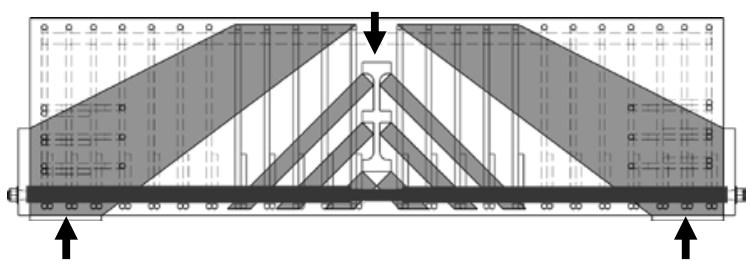

Fig. 10. Initial model invalidated by the test.

\section{Interpretation of the results and new model}

The original model was based on an engineering approach with two major hypotheses :

- An inclination of $45^{\circ}$ with respect to the vertical was considered for the diagonal struts;

- The lower bound theorem of plasticity was supposed applicable, and a uniform distribution of the forces in the different stirrups was considered.

It has been shown that these two hypotheses are not verified, and lead to an unsafe estimation of the stresses in the stirrups at collapse. It is thus mandatory to reconsider the definition of the strut-and-tie model.

The strut-and-tie method is supposed to rely on the lower bound theorem : a stress field that satisfies equilibrium and does not violate yield criteria at any point provides a lower-bound estimate of capacity of elements made of elasticperfectly plastic materials. 
However the usual references for this method $[1,2,3]$ don't take it as a start and are more careful for the definition of the strut-and-tie model. They base it on the stress fields obtained by the theory of elasticity. Compressive principal stresses are aggregated in struts, while tensile stresses are condensed in ties. This implies a reorientation of forces to take into account that rebars (and ties) will be often placed in orthogonal directions that don't correspond exactly to tensile principal stresses.

This method, as pointed out by Schlaich [1], obviously neglects some ultimate load capacity. But it limits the ductility demand at design load. Our results show this lack of ductility, as the full yield force of the five stirrups cannot be mobilized. As a consequence, the test specimen $\mathrm{CS}-\mathrm{H}+\mathrm{R}$ is studied in the following by linear elastic analysis and stress trajectories are drawn using FINELG [4]. A total vertical force of 1668 $\mathrm{kN}$, the mean of the maximum experimental loads, is applied together with a horizontal force of $1000 \mathrm{kN}$, the mean of the horizontal loads. Results are presented on Fig. 11. The position of the stirrups and longitudinal bars is marked by black lines. This model shows clearly that struts are not parallel, and that their inclination varies with the level of the load in the beam.

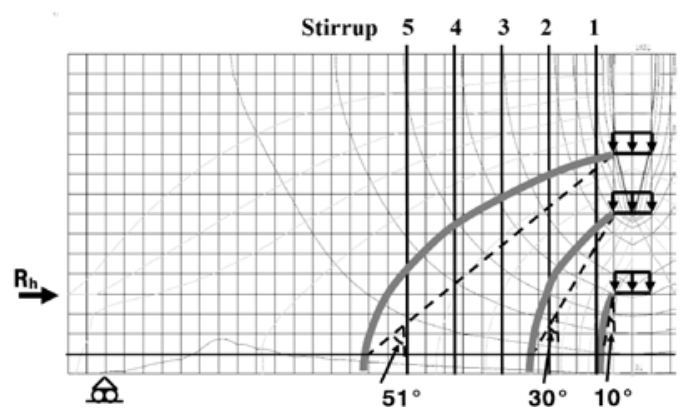

Fig. 11. Elastic stress trajectories $-\mathrm{CSH}-\mathrm{R}+3$.

A new strut and tie model is thus drawn, see Fig. 12. The fifth stirrup is not considered in order to be conform with experimental results.

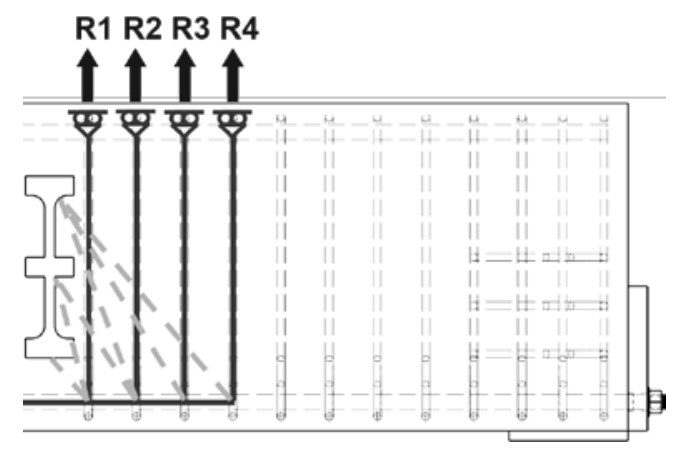

Fig. 12. New strut and tie model.
The evolution of the forces in the stirrups are compared to the experimental results on Fig. 13. The correlation is good.

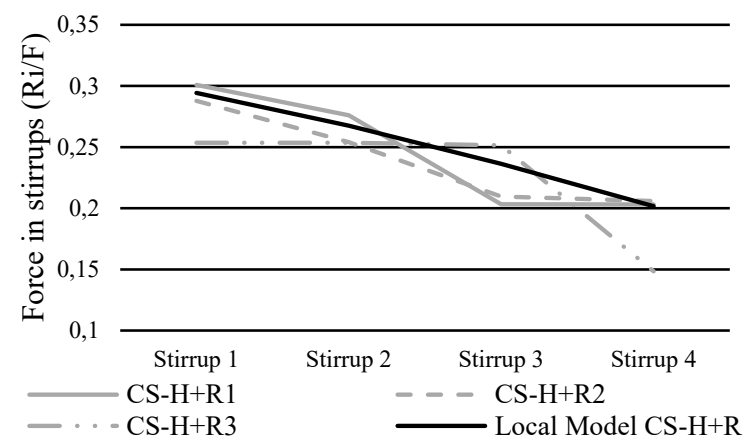

Fig. 13. Comparison of forces in the stirrups.

The fact that the fifth stirrup is not mobilized can be explained by two reasons :

- First of all the real strut, that can be visualized by the cracking, is more straight than elastic stress trajectories and corresponds to the secant line drawn in dotted line on Fig. 11. As a consequence, the anchoring length of the stirrup in the strut is not large enough to develop its yielding;

- A simple analytical reasoning shows that the force in the stirrups varies with $\cos ^{3} \alpha$, with $\alpha$ the inclination on the vertical of the strut, see Fig. 14 and Eqs (1), (2) :

$$
\begin{aligned}
N_{\text {strut }}= & E A \frac{\Delta L}{L}=\frac{E A_{\text {strut }} \delta \cos (\alpha)}{d / \cos (\alpha)} \\
N_{\text {stirrup }} & =\mathrm{N}_{\text {strut }} \cos (\alpha) \\
& =\frac{E A_{\text {strut }}}{d} \delta \cos ^{3}(\alpha)
\end{aligned}
$$

With $A_{\text {strut }}$ the section of the strut, $\delta$ the vertical displacement of the point of application of the load, $\alpha$ the inclination of the strut on the vertical, $d$ the distance from the load to the lower longitudinal rebar.

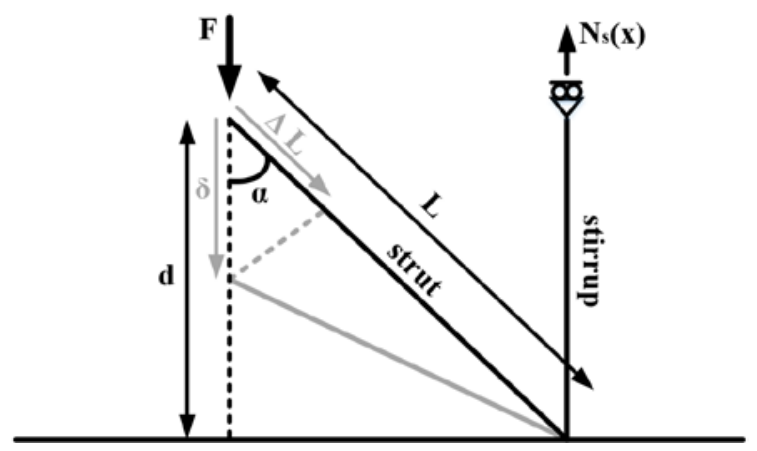

Fig. 14. Effect of the inclination of the strut on the force in the stirrup. 
As a consequence, the force in the stirrups in the elastic model decrease rapidly with the distance, and the force in the fifth stirrup is negligible.

As a conclusion, it appears clearly that the strut-and-tie design model must be carefully drawn from elastic stress trajectories, and that the inclination of the struts bounding the loads to the suspending stirrups plays a key role in the distribution of the forces in the different stirrups, by limiting the contribution of the further stirrups.

\section{General model}

Before defining a general model, a point must still be investigated. Elastic stress trajectories in Fig. 11 show that the level of application of the load modifies substantially the angle of diffusion. It is very low when the load is applied near the lower flange of the concrete beam, and even when the load is applied at mid height it is still limited to $30^{\circ}$. This limits the number of the stirrups that can support the steel profile.

A parametrical study has been made in order to get general information on the evolution of the angle of diffusion with the level of application of the load. Several beams with different lengths have been computed by elastic linear analysis, and the angle of diffusion has been deduced from the stress trajectories. As can be seen on Fig. 15, the angle can be as low as $10^{\circ}$ when the load is applied in the lower half of the specimen.

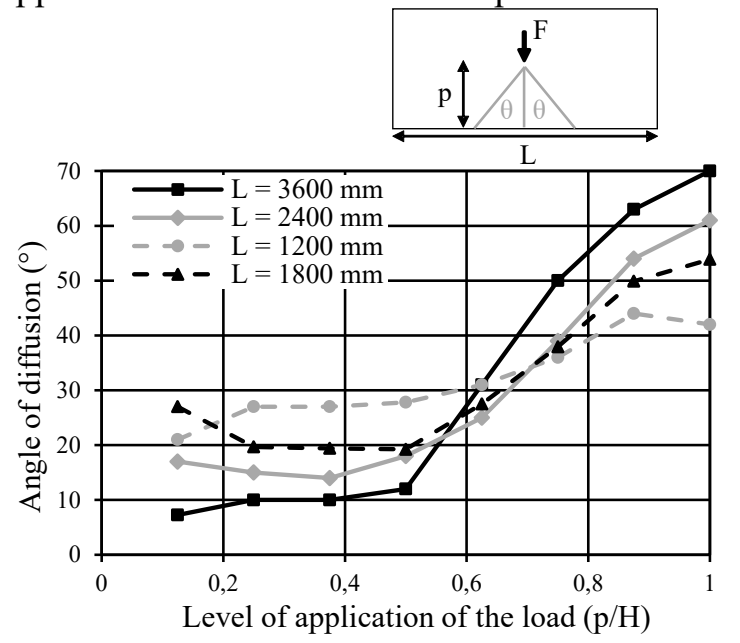

Fig. 15. Determination of the total force in the stirrups.

However the elastic stress trajectories must not be considered as exact and a variation of $15^{\circ}$ can be adopted [1]. As a consequence, it is proposed to adopt an angle of $18^{\circ}$. It is the angle that is proposed in EC2 [5] for the case of a secondary concrete beam, in the $\S 9.2 .5$ : indirect support. If the lower flange of the steel beam is upper than $0.7 h_{c}$, it is proposed to open the angle up to $30^{\circ}$.

This angle may seem quite little. It is fixed to conserve a rather good efficiency of all the stirrups within the diffusion zone. Indeed, the force in the stirrups located within the angle of diffusion cannot be considered as uniform, as has been shown by experimental evidence. The ultimate load is then defined by the yielding of the first stirrup. The reduction factor $\rho$ of the yielding force of the stirrups considered for the support of the steel profile can be computed supposing that the stirrups can be replaced by a tie distributed uniformly along the length of the beam, see Fig. 16. The force can be computed as the integral of the forces in the distributed stirrup :

$\frac{F}{2}=\int_{x_{i}}^{x_{f}} N_{S}(x) d x=\int_{x_{i}}^{x_{f}} a_{S} f_{y} \frac{\cos ^{3} \alpha(x)}{\cos ^{3} \alpha_{i}(x)} d x$

That gives

$F=\rho A_{s} f_{y}$

with

$\rho=\frac{1}{x_{f}-x_{i}} \int_{x_{i}}^{x_{f}} \frac{\cos ^{3} \alpha(x)}{\cos ^{3} \alpha_{i}(x)} d x$

With $A_{s}$ the total section of the stirrups within the angle of diffusion, $a_{s}$ the distributed section, $\mathrm{x}_{\mathrm{i}}$ the abscissa of the beginning of the zone of diffusion at the level of the longitudinal rebar, $\mathrm{x}_{\mathrm{f}}$ the abscissa at the end of the angle of diffusion, and $\alpha_{i}$ the angle of the strut the more inclined in the diffusion zone.

Considering $x_{i}=0$, the evolution of $\rho$ with the maximum angle of diffusion has been drawn in Fig. 17. As can be seen, the reduction factor for an angle of diffusion of $30^{\circ}$ is around 0.9 .

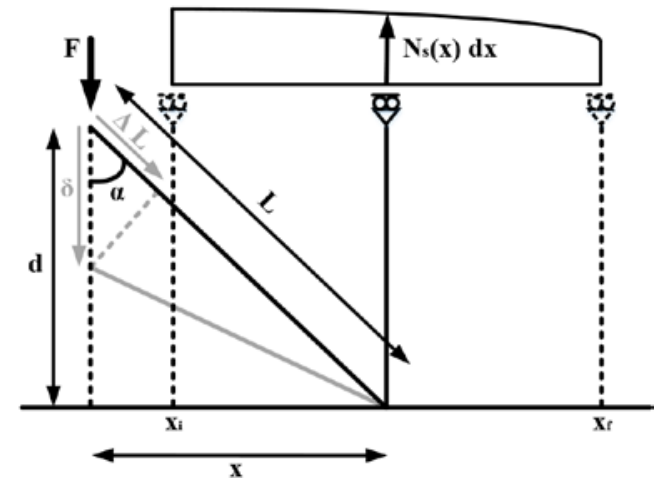

Fig. 16. Determination of the total force in the stirrups. 


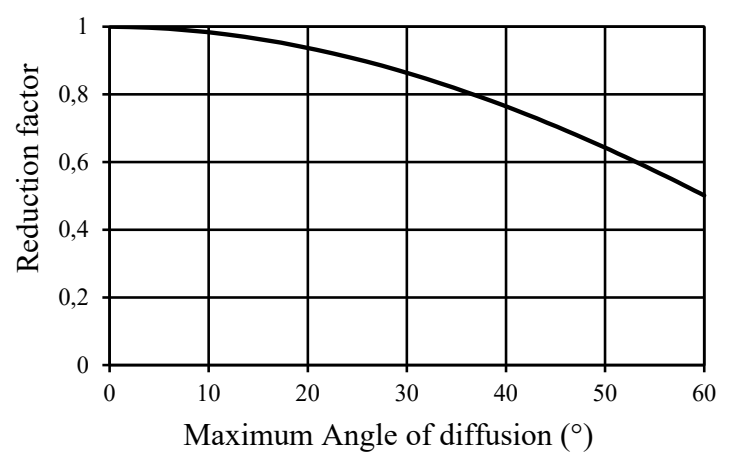

Fig. 17. Evolution of the reduction factor of the stirrup resistance with the angle of diffusion.

\section{Conclusions}

In this paper, an experimental campaign investigating the support of steel beams by concrete beams by simple embedment has been presented. It has been shown that the level of the steel beam within the section of the concrete beam modifies substantially the angle of diffusion of the reaction within the concrete. Furthermore it has been shown that the stresses in the stirrups within this angle of diffusion cannot be considered as uniform if this angle is large.

As a consequence, the strut-and-tie model presented Figs. 18 and 19 is proposed for the design, with the following recommendations, that have been added to the SMARTCOCO design guide [6] :

(1) The inclination of the struts is limited to $\tan (\theta) \leq 0.3\left(\theta<17^{\circ}\right)$.

(2) If the height of the upper flange $\mathrm{Z}_{\mathrm{uf}}$ is larger than $0.7 \mathrm{~h}_{\mathrm{c}}, \mathrm{h}_{\mathrm{c} \text { being }}$ the height of the concrete beam, then the inclination of the struts may be increased up to $\tan (\theta) \leq 0.6\left(\theta<30^{\circ}\right)$.

(3) In this latter case, the resistance of the ties $\mathrm{F}_{\text {td }}$ must be reduced by a factor 0.9 to take into account the non-uniform distribution of the stresses in the different stirrups.

(4) The inclination of the struts depends largely on the general configuration of the steel and concrete beams. The minimal values given in (2) and (3) can be increased on the basis of the stress trajectories obtained with a linear elastic analysis. In the model, each stirrup must then be linked by an independent strut to the steel profile in order to take into account the non-uniform distribution of the normal force in the different stirrups.

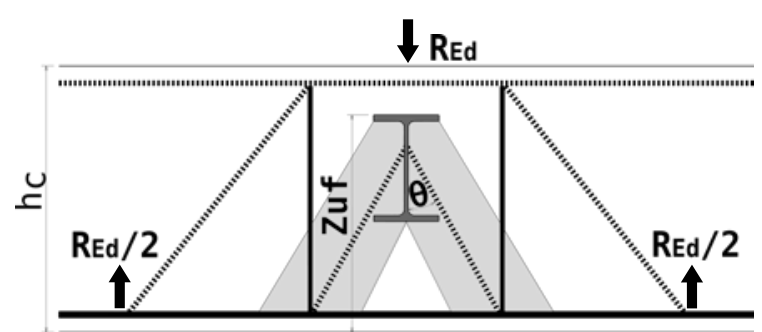

Fig. 18. Strut and tie model for the indirect support of a steel beam inside a concrete beam.

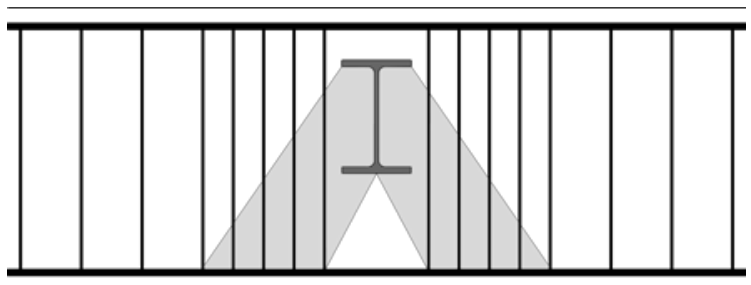

Fig. 19. Arrangement of reinforcement for the indirect support of a steel beam inside a concrete beam.

Acknowledgements. This paper was developed in the frame of the SMARTCOCO project funded by RFCS, the Research Fund for Coal and Steel of the European Commission, Research grant agreement RFSR-CT-201200031 Smartcoco. The companies BESIX and ArcelorMittal are also acknowledged for their involvement in the project.

\section{References}

[1] Schlaich J, Schafer K, Jennewein M. Toward a consistent design of structural concrete. PCI Journal 1987, 32:3.

[2] Fib. Structural concrete : textbook on behaviour, design and performance. Updated knowledge of the CEB/FIP Model Code 1990. fib Bulletin No. $3 ; 2011$.

[3] Fib. Design examples for strut-and-tie models. fib Bulletin No. 61; 2011.

[4] de Ville V, Somja H, Pesesse C. Finelg user's manual, version 9.1.; 2016.

[5] CEN2004. Eurocode 2: EN 1992-1-1 Design of concrete structures, Part 1.1 - General Rules for buildings. European Committee for Standardizations, Brussels; 2004

[6] Plumier A. SMARTCOCO design guide; 2017. 